\title{
Long-term incidence of chronic postsurgical pain after thoracic surgery for lung cancer: a 10-year single- center retrospective study
}

\author{
Susie Yoon, ${ }^{1,2}$ Won-Pyo Hong, ${ }^{1}$ Hyundeok Joo, ${ }^{3}$ Hansol Kim, ${ }^{1}$ Samina Park, ${ }^{4,5}$ \\ Jae-Hyon Bahk, ${ }^{1,2}$ Ho-Jin Lee (id) ${ }^{1,2}$
}

\begin{abstract}
- Additional material is published online only. To view please visit the journal online (http://dx.doi.org/10.1136/ rapm-2020-101292).
\end{abstract}

'Department of Anesthesiology and Pain Medicine, Seoul National University Hospital, Seoul, The Republic of Korea ${ }^{2}$ Department of Anesthesiology and Pain Medicine, Seoul National University College of Medicine, Seoul, The Republic of Korea

${ }^{3}$ Seoul National University College of Medicine, Seoul, The Republic of Korea

${ }^{4}$ Department of Thoracic and Cardiovascular Surgery, Seoul National University Hospital, Seoul, The Republic of Korea Department of Thoracic and Cardiovascular Surgery, Seoul National University College of Medicine, Seoul, The Republic of Korea

Correspondence to Dr Ho-Jin Lee, Department of Anesthesiology and Pain Medicine, Seoul National University College of Medicine, Seoul, The Republic of Korea; zenerdiode03@gmail.com

Received 9 January 2020 Revised 19 February 2020 Accepted 22 February 2020 Published Online First 17 March 2020

\begin{abstract}
Background The long-term incidence of chronic postsurgical pain (CPSP) after thoracic surgery has not yet been reported.

Methods We retrospectively reviewed the electronic medical records of 4218 consecutive patients who underwent thoracic surgery for lung cancer between 2007 and 2016. We evaluated the long-term incidence of CPSP after thoracic surgery at intervals of 3 months for 36 months. A Cox proportional hazard regression analysis was performed to investigate the predictors of CPSP after thoracic surgery.
\end{abstract}

Results A total of 3200 patients were included in the analysis. Of these, $459(14.3 \%)$ and $558(17.4 \%)$ patients were diagnosed with CPSP within 3 and 36 months after surgery, respectively. Furthermore, the incidence of CPSP decreased over time. Additionally, 99 (3.1\%) patients were newly diagnosed with CPSP at least 6 months after surgery. Female sex (HR 1.20, 95\% $\mathrm{Cl} 1.00$ to $1.43 ; p=0.04$ ), longer duration of surgery (HR $1.11,95 \% \mathrm{Cl} 1.03$ to $1.20 ; p<0.01$ ), higher 11-point Numeric Rating Scale score at first outpatient visit after surgery (HR $1.29,95 \% \mathrm{Cl} 1.24$ to $1.34 ; \mathrm{p}<0.001$ ), postoperative chemotherapy (HR $1.55,95 \% \mathrm{Cl} 1.26$ to $1.90 ; p<0.001$ ), and postoperative radiation therapy (HR $1.35,95 \% \mathrm{Cl} 1.05$ to $1.74 ; p=0.02$ ) were significant predictors of CPSP for 36 months after surgery. Conclusion Our study showed a decreasing trend in the incidence of CPSP as well as delayed-onset or recurrent CPSP after thoracic surgery. A better understanding of the progression of CPSP after thoracic surgery may provide important information on its prediction and treatment.

\section{INTRODUCTION}

Chronic postsurgical pain (CPSP) refers to pain that persists beyond the expected recovery period in the area associated with surgery. ${ }^{12}$ Its incidence has been reported to be $3 \%-85 \%$, and varies greatly depending on the type of surgery or the definition of CPSP. ${ }^{3}$ The risk factors for CPSP include intraoperative nerve injury, surgery duration, pre-existing pain, younger age, female sex, genetic predisposition, psychological vulnerability, and severity of acute postoperative pain. ${ }^{4}$ CPSP has been associated with functional disability, poor recovery, and impaired quality of life. ${ }^{5}$ It has also been associated with prolonged opioid use after surgery, which leads to opioid-induced adverse events. ${ }^{6}$
CPSP is a relatively common complication after thoracic surgery, with an incidence rate as high as $65 \% .^{3} \mathrm{~A}$ meta-analysis of prospective studies reported that the incidence of CPSP at 3 and 6 months after thoracotomy was $57 \%$ and $47 \%$, respectively. ${ }^{7}$ Although the introduction of videoassisted thoracic surgery (VATS) has resulted in minimal incision sites and decreased acute postoperative pain, CPSP remains an important postoperative complication in patients after thoracic surgery. ${ }^{8}$ In addition, as early-stage lung cancer detection has increased with the introduction of screening tests, lung surgery, which is a standard treatment for early-stage lung cancer, has been increasingly performed. ${ }^{9}$ The early diagnosis and treatment for lung cancer have led to an increase in the survival rate among patients with lung cancer. ${ }^{10}$ Therefore, CPSP related to the quality of life after surgery has become an important issue for patients with lung cancer. ${ }^{11}$

According to the recently proposed updated criteria of CPSP, it could either be a continuation of acute postoperative pain or pain that occurs after an asymptomatic period. ${ }^{1}$ However, most previous studies have reported point incidence rather than a long-term trend. Therefore, we investigated the long-term incidence of CPSP after thoracic surgery with respect to delayed-onset and recurrent CPSP. To this end, we investigated the trend of CPSP incidence after thoracic surgery at intervals of 3 months for 36 months. A multivariable Cox proportional hazard regression analysis was performed to investigate the perioperative predictors of CPSP after thoracic surgery.

\section{METHODS}

The requirement for informed consent was waived due to the retrospective design of this study.

We retrospectively reviewed the electronic medical records of patients aged $\geq 18$ years who underwent thoracic surgery for lung cancer at our hospital between January 2007 and December 2016. Data of all patients who satisfied the above conditions during the study period were collected without an a priori sample size calculation. During the 10-year study period, 4218 adult patients underwent lung surgery at our hospital. The exclusion criteria were as follows: (1) patients with previous thoracic surgery $(n=47) ;(2)$ those who underwent reoperation at the ipsilateral site during the study period ( $n=134)$; (3) those who underwent surgical 
methods other than VATS and thoracotomy (sternotomy, $\mathrm{n}=8$; clamshell incision, $\mathrm{n}=2$ ); (4) those who were not followed up for at least 3 months after surgery; and (5) those with inadequate medical records related to pain intensity $(\mathrm{n}=827)$. The remaining 3200 patients were finally analyzed.

Data on demographics, medical history, anesthesia/surgeryrelated parameters, postoperative pain-related parameters, and adjuvant chemotherapy and radiation therapy, which have been associated with chronic postoperative pain, were collected from the Seoul National University Hospital Patients Research Environment (SUPREME) system (table 1). ${ }^{4}$ We investigated whether the patients underwent the intraoperative intercostal nerve blockade or percutaneous wound injection based on the electronic medical records documented by the surgeons.

Preoperative administrations of oral analgesics and antipsychotics were investigated instead of pre-existing chronic pain and psychiatric diseases, respectively, due to the lack of detailed information about these diseases. We also investigated the treatments that the patients received for CPSP during the study period. The medication included strong opioids (morphine, oxycodone, hydrocodone, hydromorphone, or transdermal fentanyl patch), weak opioids (tramadol or a tramadol/acetaminophen combination), non-steroidal anti-inflammatory agents, acetaminophen, antidepressants (tricyclic antidepressants or serotonin-norepinephrine reuptake inhibitors), anticonvulsants (gabapentin or pregabalin), and transdermal lidocaine patch. Prolonged opioid use after surgery was defined as one or more strong opioid prescriptions for more than 3 months after surgery. ${ }^{12}$ We did not attempt to estimate missing data. All patients, including those with missing values, were included in the analyses with the available data.

Patients received total intravenous anesthesia consisting of propofol and remifentanil, or inhalational anesthesia with volatile agent and supplementary opioids. The type of anesthesia was selected based on the preference of the anesthesiologist. Intravenous patient-controlled analgesia consisted of a combination of fentanyl $(10-20 \mu \mathrm{g} / \mathrm{mL})$ and morphine $(0.4-0.7 \mathrm{mg} / \mathrm{mL})$ at a continuous infusion rate of $0.5 \mathrm{~mL} /$ hour (intravenous morphine equivalent dose (IVME) $0.5-1 \mathrm{mg} /$ hour) and a bolus of $1 \mathrm{~mL}$ (IVME 1-2 mg) with a lockout interval of $10 \mathrm{~min}$. Generally, when patients tolerated oral nutrition, oral pain medications were initiated. For open thoracotomy, unless contraindicated, a thoracic epidural or paravertebral catheter was placed at approximately T5-T6, and $0.15 \%$ ropivacaine and fentanyl $(2 \mu \mathrm{g} / \mathrm{mL})$ were infused at $2-4 \mathrm{~mL} /$ hour as tolerated, based on blood pressure and pain scores.

VATS was performed by a three-port technique consisting of three incisions (less than $5 \mathrm{~cm}$ ) with rib sparing. Posterolateral thoracotomy was performed through the fourth or fifth interspace by making $20 \mathrm{~cm}$ incisions, sparing the serratus anterior muscle. The surgical procedure was chosen by the attending thoracic surgeon based on clinical features including patient age, comorbid disease, pulmonary function, and tumor characteristics. Intercostal nerve blockade or percutaneous wound injection using $0.25 \%$ bupivacaine was performed before or after the main surgical procedure according to the attending surgeons.

CPSP was defined when the pain intensity measured by an 11-point Numeric Rating Scale (NRS) was higher than 3 points for at least 3 months during the 36 months after surgery. ${ }^{13}$ We used the pain intensity of a surgical site based on the electronic medical records. In the outpatient clinic, patients were asked to rate their current pain intensity along the scar using the 11-point NRS (0: no pain at all, 10: worst imaginable pain) by thoracic surgeons. CPSP was determined as consecutive NRS scores
Table 1 Characteristics and perioperative parameters between patients with and without chronic postsurgical pain after thoracic surgery

\begin{tabular}{|c|c|c|c|}
\hline & CPSP group & Non-CPSP group & P value* ${ }^{*}$ \\
\hline Sample size, $\mathrm{n}$ & 558 & 2642 & \\
\hline \multicolumn{4}{|l|}{ Demographic characteristics } \\
\hline Age, years & $64(58-70)$ & $64(56-71)$ & 0.82 \\
\hline Sex & & & 0.09 \\
\hline Male & $295(52.9)$ & $1503(56.9)$ & \\
\hline Female & $263(47.1)$ & 1139 & \\
\hline Body mass index, $\mathrm{kg} / \mathrm{m}^{2}$ & $23.7(21.9-25.8)$ & $23.6(21.6-25.6)$ & 0.32 \\
\hline Current smoker & $151(27.1)$ & $761(28.8)$ & 0.44 \\
\hline \multicolumn{4}{|l|}{ Background medical status } \\
\hline ASA class $1 / / I / / 111$ & $160(28.7) / 364(65.2) / 34(6.1)$ & $\begin{array}{l}752(28.5) / 1749 \\
(66.2) / 141(5.3)\end{array}$ & 0.76 \\
\hline Diabetes mellitus & $82(14.7)$ & $375(14.2)$ & 0.81 \\
\hline History of tuberculosis & $45(8.1)$ & $202(7.6)$ & 0.80 \\
\hline Preoperative analgesic use & $24(4.3)$ & $110(4.2)$ & 0.98 \\
\hline Preoperative strong opioid use $\ddagger$ & $0(0)$ & $6(0.2)$ & 0.56 \\
\hline Preoperative antipsychotics use & $8(1.4)$ & $49(1.9)$ & 0.61 \\
\hline Preoperative chemotherapy & $19(3.4)$ & $84(3.2)$ & 0.89 \\
\hline Postoperative chemotherapy & $196(35.1)$ & $667(25.2)$ & $<0.001$ \\
\hline Preoperative radiation therapy & $12(2.2)$ & $60(2.3)$ & 0.99 \\
\hline Postoperative radiation therapy & $85(15.2)$ & $276(10.4)$ & $<0.01$ \\
\hline Previous other surgeries & $358(64.2)$ & $1678(63.5)$ & 0.81 \\
\hline \multicolumn{4}{|l|}{ Surgical characteristics } \\
\hline Operation year, $\mathrm{n}$ & & & $<0.01$ \\
\hline $\begin{array}{l}\text { 2007/2008/2009/2010/2011/2012/ } \\
2013 / 2014 / 2015 / 2016\end{array}$ & $\begin{array}{l}45 / 33 / 24 / 38 / 71 / 66 / 66 / \\
78 / 75 / 62\end{array}$ & $\begin{array}{l}122 / 119 / 141 / 147 / \\
268 / 331 / 319 / 364 / \\
394 / 437\end{array}$ & \\
\hline Operative technique & & & $<0.001$ \\
\hline Thoracotomy & $193(34.6)$ & $611(23.1)$ & \\
\hline VATS & $365(65.4)$ & $2031(76.9)$ & \\
\hline Operation type & & & 0.03 \\
\hline Pneumonectomy & $16(2.9)$ & $54(2.0)$ & \\
\hline Lobectomy & $471(84.4)$ & $2117(80.1)$ & \\
\hline Segmentectomy & $28(5.0)$ & $213(8.1)$ & \\
\hline Wedge resection & $39(7.0)$ & $244(9.2)$ & \\
\hline Biopsy & $3(0.5)$ & $13(0.5)$ & \\
\hline Explorative surgery & $1(0.2)$ & $1(0)$ & \\
\hline Operation time, min & $160(130-215)$ & $147(120-190)$ & $<0.001$ \\
\hline Type of anesthesia & & & 0.02 \\
\hline Inhalation agent & $187(33.5)$ & $748(28.3)$ & \\
\hline TIVA & $371(66.5)$ & $1894(71.7)$ & \\
\hline Type of PCA & & & 0.15 \\
\hline Intravenous PCA & $426(76.3)$ & $2065(78.2)$ & \\
\hline Epidural PCA & $129(23.1)$ & $551(20.9)$ & \\
\hline Paravertebral PCA & $3(0.5)$ & $9(0.3)$ & \\
\hline None & $0(0.0)$ & $17(0.6)$ & \\
\hline Other analgesic techniques & & & 0.04 \\
\hline Intercostal nerve blockade & $81(14.5)$ & $493(18.7)$ & \\
\hline Percutaneous wound injection & $62(11.1)$ & $241(9.1)$ & \\
\hline Maximal NRS score during POD 2, 0-10 & $7(5-8)$ & $7(5-8)$ & 0.02 \\
\hline $\begin{array}{l}\text { NRS score at first outpatient visit after } \\
\text { surgery, } 0-10\end{array}$ & $3(3-5)$ & $2(0-3)$ & $<0.001$ \\
\hline
\end{tabular}

The values are presented as median (IQR) or number (\%).

P value compares CPSP group versus non-CPSP group.

Continuous variables were compared using Mann-Whitney $U$ test. Proportions were compared using $\chi^{2}$ or Fisher's exact test according to their expected counts.

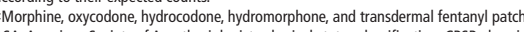

Anesthesiologists physical status classification; CPSP, chronic postsurgical pain; NRS, Numeric Rating Scale; PCA, patient-controlled analgesia; POD, postoperative day; TIVA, total intravenous anesthesia; VATS, video-assisted thoracic surgery

$\geq 3$ in two measurements at least 3 months apart. ${ }^{13} 14$ Exceptionally, CPSP at 3 months after surgery was defined using the NRS scores measured at a first outpatient visit and at 3-month follow-up after surgery, where the time interval between the two points was less than 3 months. The first outpatient follow-up was performed approximately 2 weeks after surgery. We classified patients into the following four groups based on their condition at 3-month intervals for 36 months after surgery: newly developed CPSP, continued CPSP, recurrent CPSP, non-CPSP, and lost 


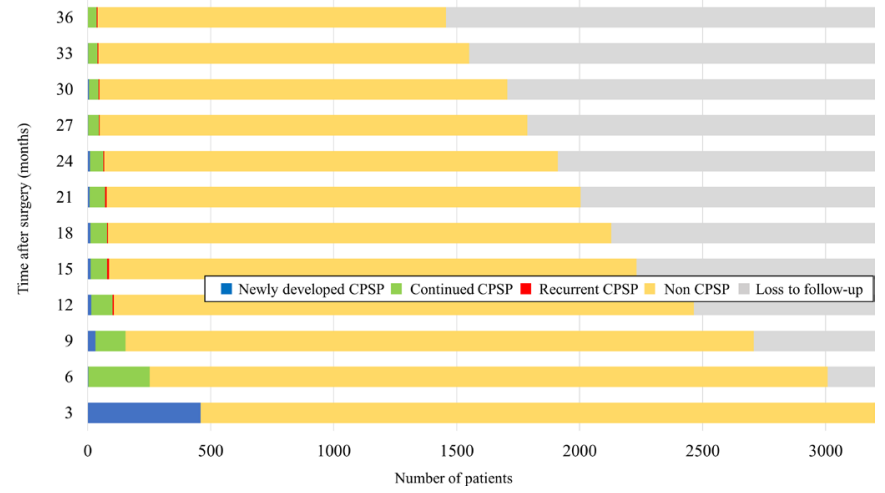

Figure 1 Long-term incidence of chronic postsurgical pain (CPSP) during the first 36 months after thoracic surgery in all cohorts.

to follow-up. The duration of CPSP was calculated at 3-month intervals. In the event of a recurrent CPSP, the summed duration of CPSP within 36 months after surgery was used.

\section{Statistical analysis}

R V.3.6.1 (R Foundation for Statistical Computing, Vienna, Austria) was used to analyze the data. A two-sided $p<0.05$ was considered statistically significant for all analyses. The ShapiroWilk test and the quantile-quantile plot were used to determine the normal distribution of continuous variables. Continuous data are reported as mean and SD or median and IQR, and were compared between groups using the independent t-test or Mann-Whitney U test according to the normality of data. Categorical data are described as frequency or percentage and were compared between groups using the $\chi^{2}$ test or Fisher's exact test according to their expected counts. We compared the groups based on the presence/absence of CPSP (CPSP vs non-CPSP) as well as on the onset of CPSP (delayed-onset CPSP vs acute-onset CPSP). Delayed-onset CPSP was defined when the CPSP was not diagnosed at 3 months after surgery but was later diagnosed. Since a relatively large proportion of patients with missing data were excluded, we also compared these excluded patients with the patients included in the study.

To investigate the association between perioperative parameters and the risk of CPSP, we conducted a Cox proportional hazard regression analysis for chronic postoperative pain during the 36 months after surgery. The proportional hazard assumptions of the Cox regression analysis were tested by visual inspection of log-minus-log survival plots for categorical variables and

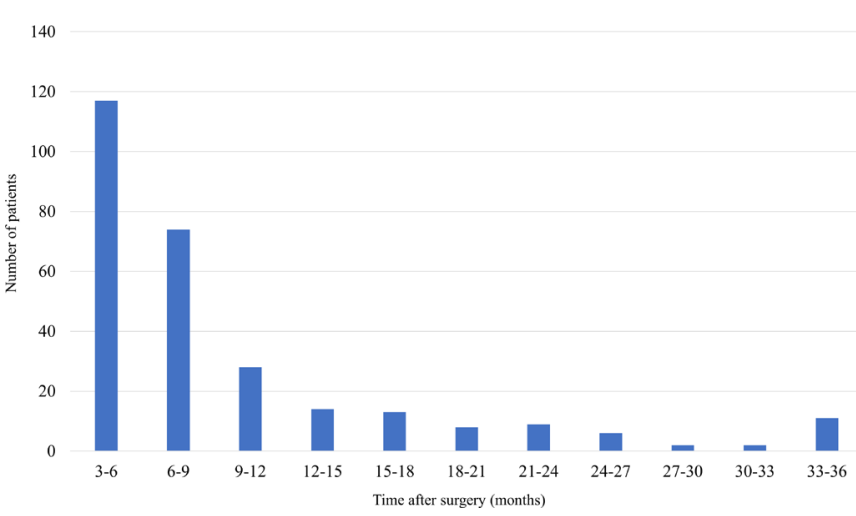

Figure 2 Summed duration of chronic postsurgical pain (CPSP) in patients with CPSP followed up for 36 months after surgery (total, $n=1457 ;$ CPSP, $n=284$ ). restricted cubic splines for continuous variables. The following variables were included in the analyses without the variable selection process: age, female sex, preoperative analgesic use, thoracotomy (vs VATS), operation time (hour), NRS score at first outpatient visit after surgery, postoperative chemotherapy, postoperative radiation therapy, and year of surgery. We used the NRS score at a first outpatient visit after surgery instead of the maximal NRS score on postoperative day 2 as a variable representing the intensity of acute postoperative pain. During hospitalization, several ward nurses evaluated the pain intensity on a rotational basis, and the evaluation method was not uniform. We believe that NRS evaluated by surgeons in the outpatient visit can better differentiate the degree of acute postoperative pain. We included the year of surgery due to concerns regarding the recent reduction in the frequency of CPSP. The following variables were not included in the analysis due to the violation of proportional hazard assumptions: current smoker, diabetes mellitus, main anesthetic agents, preoperative antipsychotics use, intraoperative intercostal nerve blockade, and percutaneous wound injection.

\section{RESULTS}

Among the 3200 patients in this study, 558 (17.4\%, 95\% CI $16.2 \%$ to $18.8 \%$ ) were diagnosed with CPSP within 36 months after surgery. Patients' characteristics and perioperative parameters are summarized in table 1 . Patients with CPSP were more likely to receive postoperative chemotherapy or radiation therapy, more likely to receive thoracotomy, had a longer duration of surgery, more likely to receive inhalation anesthetic agents during surgery, less likely to receive intraoperative intercostal nerve blockade, and had higher NRS score at the first outpatient visit after surgery. No patients with CPSP had taken any opioid medications before their surgery. The characteristics and perioperative parameters between patients included and excluded in the study due to loss of follow-up or inadequate medical records are presented in online supplementary table S1. The mortality within 36 months after surgery was significantly higher in the excluded group than in the included group $(n=297,9.3 \%$ vs $\mathrm{n}=263,31.8 \% ; \mathrm{p}<0.001)$. Among the excluded patients, 57 (6.9\%) died within 3 months after surgery.

Figure 1 and online supplementary table S2 show the trend in the status of chronic pain at 3-month intervals during the 36 months after thoracic surgery. Overall, 459 (14.3\%, 95\% CI $13.2 \%$ to $15.6 \%)$ patients had CPSP at 3 months after surgery, and $99(3.1 \%, 95 \%$ CI $2.5 \%$ to $3.8 \%)$ were additionally diagnosed with CPSP at least 6 months after surgery. Patients' clinical characteristics and perioperative parameters according to the onset of CPSP are presented in online supplementary table S3. The incidence of CPSP tended to decrease over time after surgery. Furthermore, 40 of 1457 (2.7\%, 95\% CI 2.0\% to 3.7\%) patients were diagnosed with CPSP at 36 months after surgery. A total of 1457 patients were followed up for 36 months after surgery, of whom 284 were diagnosed with CPSP. The median (IQR) of their summed duration of CPSP was 6 (3-9) months (figure 2). Among them, 26 patients showed improvement in CPSP, followed by relapse. The incidence of CPSP at 3 months after surgery did not show significant differences between the years of surgery $(p=0.38)$ (figure 3$)$. During the study period, there were changes in perioperative parameters in our hospital (online supplementary table S4).

Among the 558 patients with CPSP, 475 (85.1\%, 95\% CI $82.2 \%$ to $88.1 \%$ ) received analgesics for CPSP after surgery (table 2). The most commonly prescribed analgesics were weak 


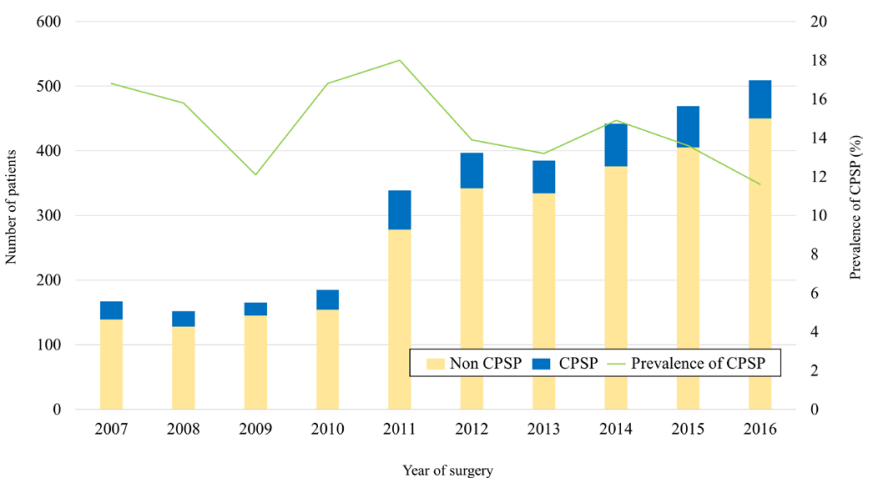

Figure 3 Trend in the incidence of chronic postsurgical pain (CPSP) at 3 months after thoracic surgery according to year of surgery in all cohorts.

opioids such as tramadol or tramadol/acetaminophen combination $(81.5 \%, 95 \%$ CI $78.1 \%$ to $84.5 \%)$. Furthermore, 206 (36.9\%, 95\% CI $33.5 \%$ to $41.5 \%)$ patients received strong opioids, and $93(17.0 \%$, 95\% CI $13.6 \%$ to $19.8 \%)$ received strong opioids for more than 90 days after surgery. Thirty-six $(6.5 \%, 95 \%$ CI $4.7 \%$ to $8.8 \%)$ patients were referred to the pain center of our department for CPSP.

\begin{tabular}{|c|c|}
\hline Treatments for chronic postsurgical pain & $\mathrm{n}=558$ \\
\hline No medication & $83(14.9)$ \\
\hline Acetaminophen & $131(23.5)$ \\
\hline Time from surgery to first prescription (months) ${ }^{*}$ & $8(4-18)$ \\
\hline Duration of prescription (months)* & $0(0-5)$ \\
\hline Anticonvulsantst & $71(12.7)$ \\
\hline Time from surgery to first prescription (months)* & $8(3-16)$ \\
\hline Duration of prescription (months)* & $4(1-14)$ \\
\hline Antidepressants $\ddagger$ & $38(6.8)$ \\
\hline Time from surgery to first prescription (months)* & $9(3-21)$ \\
\hline Duration of prescription (months)* & $6(0-15)$ \\
\hline Lidocaine transdermal patch & $24(4.3)$ \\
\hline Time from surgery to first prescription (months)* & $7(3-13)$ \\
\hline Duration of prescription (months)* & $1(0-7)$ \\
\hline Non-steroidal anti-inflammatory drugs & $140(25.1)$ \\
\hline Time from surgery to first prescription (months) ${ }^{*}$ & $4(2-13)$ \\
\hline Duration of prescription (months)* & $0(0-4)$ \\
\hline Weak opioids§ & $455(81.5)$ \\
\hline Time from surgery to first prescription (months)* & $1(1-1)$ \\
\hline Duration of prescription (months) ${ }^{*}$ & $0(0-1)$ \\
\hline Strong opioids $\mathbb{1}$ & $206(36.9)$ \\
\hline Time from surgery to first prescription (months) * $^{*}$ & $4(1-14)$ \\
\hline Duration of prescription (months)* & $2(0-11)$ \\
\hline Prolonged strong opioid use ( $\geq 3$ months) & $93(16.7)$ \\
\hline Refer to the pain center & $36(6.5)$ \\
\hline Fluoroscopy-guided thoracic epidural block & $19(3.4)$ \\
\hline Ultrasound-guided intercostal nerve block & $13(2.8)$ \\
\hline $10 \%$ lidocaine wound injection & $5(0.9)$ \\
\hline Pectoral nerve block & $2(0.4)$ \\
\hline
\end{tabular}

The values are presented as median (IQR) or number (\%).

* The values were rounded to the nearest month. Prescription within 2 weeks is recorded as 0 .

tGabapentin and pregabalin.

‡Tricyclic antidepressants and serotonin-norepinephrine reuptake inhibitors.

$\S$ Tramadol and tramadol/acetaminophen combination.

१Morphine, oxycodone, hydrocodone, hydromorphone, and transdermal fentanyl patch.
Table 3 Cox proportional hazard regression analysis to predict chronic postsurgical pain during the first 36 months after thoracic surgery

\begin{tabular}{|c|c|c|}
\hline & HR $(95 \% \mathrm{Cl})$ & $P$ value* \\
\hline Age & 1.01 (1.00 to 1.02 ) & 0.23 \\
\hline \multicolumn{3}{|l|}{ Sex } \\
\hline Male & Reference & \\
\hline Female & 1.20 (1.00 to 1.43$)$ & 0.04 \\
\hline Preoperative analgesic use & 1.00 (0.66 to 1.51$)$ & 0.99 \\
\hline \multicolumn{3}{|l|}{ Operative technique } \\
\hline VATS & Reference & \\
\hline Thoracotomy & 1.02 (0.82 to 1.28$)$ & 0.84 \\
\hline Operation time, hours & 1.11 (1.03 to 1.20$)$ & $<0.01$ \\
\hline $\begin{array}{l}\text { NRS score at first outpatient visit } \\
\text { after surgery }\end{array}$ & 1.29 (1.25 to 1.34$)$ & $<0.001$ \\
\hline Postoperative chemotherapy & 1.55 (1.26 to 1.90$)$ & $<0.001$ \\
\hline Postoperative radiation therapy & 1.35 (1.05 to 1.74$)$ & 0.02 \\
\hline \multicolumn{3}{|l|}{ Year of surgery } \\
\hline 2007 & Reference & \\
\hline 2008 & 0.77 (0.49 to 1.22$)$ & 0.27 \\
\hline 2009 & 0.65 (0.39 to 1.07$)$ & 0.09 \\
\hline 2010 & 1.05 (0.67 to 1.63$)$ & 0.84 \\
\hline 2011 & 1.29 (0.87 to 1.90$)$ & 0.20 \\
\hline 2012 & 0.92 (0.62 to 1.39$)$ & 0.70 \\
\hline 2013 & 0.98 (0.66 to 1.46$)$ & 0.92 \\
\hline 2014 & $0.88(0.59$ to 1.30$)$ & 0.51 \\
\hline 2015 & 0.94 (0.63 to 1.39$)$ & 0.76 \\
\hline 2016 & 1.14 (0.76 to 1.71$)$ & 0.52 \\
\hline
\end{tabular}

*Tested by Cox proportional hazards regression.

NRS, Numeric Rating Scale; VATS, video-assisted thoracic surgery.

Table 3 shows the results of the Cox proportional hazard regression analysis for CPSP during the 36 months after surgery. Female sex (HR 1.20, 95\% CI 1.00 to $1.43 ; \mathrm{p}=0.05$ ), longer surgery duration (HR 1.11, 95\% CI 1.03 to $1.20 ; \mathrm{p}<0.01$ ), higher NRS score at the first outpatient visit after surgery (HR $1.29,95 \% \mathrm{CI} 1.24$ to $1.34 ; \mathrm{p}<0.001)$, postoperative chemotherapy (HR $1.55,95 \% \mathrm{CI} 1.26$ to $1.90 ; \mathrm{p}<0.001$ ), and postoperative radiation therapy (HR 1.35, 95\% CI 1.05 to 1.74; $\mathrm{p}=0.02$ ) were significant predictors of CPSP during the first 36 months after surgery. Years of surgery were not significantly associated with CPSP.

\section{DISCUSSION}

In this study, we investigated the long-term incidence of CPSP during the first 36 months after thoracic surgery. Our study showed that the overall incidence of CPSP within 36 months after thoracic surgery was $17.4 \%$. Most CPSP were a continuation of acute postoperative pain after thoracic surgery, and the incidence of CPSP decreased over time after surgery. In Cox proportional hazard regression analyses, female sex, longer duration of surgery, higher NRS score at the first outpatient visit after surgery, postoperative chemotherapy, and postoperative radiation therapy were significant risk factors of CPSP.

In this study, the overall incidence of CPSP during the 36 months after surgery was $17.4 \%$, and the sequential incidence of CPSP showed a declining trend with time. The incidence of CPSP at postoperative 3 months was lower than that reported in most previously published studies; however, it is similar to that reported in recent retrospective multicenter study conducted in Japan. ${ }^{15}$ Only few studies have reported the long-term incidence 
of CPSP after thoracic surgery. One retrospective study reported that the incidence of CPSP at 7-12 months after thoracic surgery was $57 \%$, then declined to $21 \%$ at $6-7$ years after surgery. ${ }^{16}$ Another retrospective study reported a decreasing trend in the incidence of CPSP during the first 27 months after surgery. ${ }^{17}$ However, these studies did not investigate the serial course of CPSP in the same patient; therefore, delayed-onset or recurrent CPSP could not be described.

In our study, patients with CPSP had various recovery rates after surgery. Most CPSP continued from acute postoperative pain after surgery, but some developed after the asymptomatic period after surgery. This phenomenon has not been previously reported in patients treated by thoracic surgery but has been reported in patients with breast cancer. ${ }^{18}$ The delayed onset of pain after injury may be associated with slow anatomical, physiological, and biochemical changes, ${ }^{19}$ but the mechanism of this phenomenon remains unidentified.

Our study showed the various durations of CPSP, most of which were less than 9 months. The time concept of 'more than 3 months' used in the definition of CPSP is based on the assumption that the duration of normal tissue healing is 3 months. However, this time concept is arbitrary, and the duration of tissue healing may vary depending on multiple systemic and local factors. ${ }^{20}$ Various patient-related, surgery-related, and anesthesia-related factors affect the development and maintenance of CPSP. ${ }^{3}{ }^{4}$ Further, postoperative factors such as adjuvant therapy can also affect CPSP. This complexity may lead to a wide variety of clinical prognoses. Our study revealed that most CPSP were eventually resolved, but in some patients it persisted or recurred during the study period. Future research should consider the duration of CPSP and focus more on patients with long-term CPSP.

Our findings cast doubt on the necessity for strong opioid prescription in most patients with CPSP after thoracic surgery. Previously, opioids played a pivotal role in postoperative pain management. ${ }^{21}$ However, postsurgical opioid use can lead to prolonged opioid use, which can have a detrimental effect on patient prognosis. ${ }^{6} 122223$ Thoracic surgery is associated with a higher risk of postoperative prolonged opioid use compared with other major surgeries. ${ }^{12}$ The prescription rate of strong opioids in patients with CPSP in our study was lower than those reported in other countries. ${ }^{12} 22$ This result reflects differences in the opioid prescription pattern between countries as well as the surgical and perioperative management. ${ }^{24}$ Despite the low prescription rate of strong opioids, the rapid decline of CPSP incidence after surgery raises questions about their role in the treatment of CPSP. The prescription of strong opioids in most patients with CPSP after thoracic surgery may not be necessary. Considering the median duration of CPSP in our study, if opioids are required, physicians should prescribe opioids for only a limited period and plan for dose tapering or discontinuation at the time of the first prescription. Further studies on the benefits and risk of long-term opioids use as well as proper indication of opioids in patients with CPSP are required.

Since we considered CPSP as a time-to-event measurement, we conducted a Cox proportional hazard regression analysis rather than a binary logistic regression analysis to investigate the risk factors of CPSP. Our findings also revealed multiple risk factors, including female sex, longer duration of surgery, and higher severity of acute postoperative pain, which were associated with the development of CPSP. These results were consistent with previously published, known risk factors for CPSP. ${ }^{525}$ Although our study did not show thoracotomy as a significant risk factor for CPSP compared with VATS, there has been debate about the protective effect of VATS on the development of CPSP. $^{8} 1426$ In addition, we identified a statistically significant relationship between postoperative chemotherapy or radiation therapy and the incidence of CPSP. Although several studies have suggested that adjuvant radiotherapy or chemotherapy can increase the risk of CPSP after breast surgery, ${ }^{1827}$ the association between these therapies and CPSP after thoracic surgery has, to the best of our knowledge, not been reported. The neuropathic component accounts for a large portion of CPSP in thoracic and breast surgeries. ${ }^{28}$ Considering that radiation-induced or chemotherapy-induced neuropathies are well-known complications in patients with cancer, ${ }^{11}$ these adjuvant therapies could act as contributing factors to nerve injury associated with CPSP. However, these therapies were not found to be significant risk factors for CPSP in a recent prospective study. ${ }^{8}$ Further studies regarding how possible contributing factors to nerve injury affect the CPSP after thoracic surgery, which can be called 'double crush syndrome', are required. Although not all the risk factors showed statistically significant association with CPSP, we believe that the efforts of our physician over the past decade, such as the introduction of minimally invasive surgery, decrease of postoperative adjuvant therapies due to early diagnosis, and shorter operation time, have led to a recent decline in the incidence of CPSP.

The results of our study should be interpreted cautiously for several reasons. First, we could not include some potential predictors of CPSP because of the retrospective design of this study. We did not have detailed information regarding pre-existing pain conditions, psychological status, and prolonged chest tube drainage, which are known predictors of CPSP. ${ }^{41}$ Additionally, the genetic predisposition for chronic pain that we did not consider could also affect the occurrence of CPSP. Second, we investigated only the pain intensity of a surgical site as evaluated by NRS. Thoracic surgery can result in not only pain of a surgical site but also pain beyond the surgical site, and even other symptoms such as numbness and paresthesia. Furthermore, a large proportion of patients were excluded due to loss of follow-up or inadequate medical records. These excluded patients most likely had risk factors for CPSP reported in our study or previously published studies. We speculate that these limitations may have underestimated the incidence of CPSP in our study. However, the number of patients excluded from our study was not higher than those who declined to participate or were excluded for no clear reason in previous prospective studies. ${ }^{8} 1429$ Unlike other studies, we have tried to provide more detailed information by analyzing the characteristics of the excluded patients. Third, we could not investigate the effects of CPSP on functional disability or quality of life of patients. The impact on the quality of life of each patient can vary, even with similar severities of pain. Fourth, the number of patients lost to follow-up increased over time after surgery. Therefore, it was not possible to calculate the exact incidence of CPSP beyond 3 months after surgery. Last, there was no information regarding the treatment for CPSP received at other hospitals. However, more than $80 \%$ of opioid analgesics were prescribed at general or tertiary hospitals in Korea. ${ }^{30}$ Therefore, we hypothesized that a few patients might have been prescribed opioid analgesics at other hospitals.

\section{CONCLUSIONS}

In conclusion, our study showed various time courses of CPSP after thoracic surgery and a decreasing trend over time. A better understanding of the long-term course of CPSP may provide important information on its prediction and treatment. In 
addition, future studies should consider CPSP as a time-to-event measurement and focus on the progression of CPSP rather than its point prevalence.

Acknowledgements We would like to thank Editage (www.editage.co.kr) for English language editing.

Contributors SY: data collection, statistical analysis, manuscript preparation. W-PH: data collection, manuscript revision. HJ: data collection. HK: data collection, manuscript revision. SP: manuscript revision. J-HB: manuscript revision. HJL: study design, statistical analysis, manuscript preparation, manuscript revision.

Funding The authors have not declared a specific grant for this research from any funding agency in the public, commercial or not-for-profit sectors.

Competing interests None declared.

Patient consent for publication Not required.

Ethics approval The institutional review board (IRB) of Seoul National University Hospital approved this retrospective cohort study (IRB no 2001-005-1090). All methods were carried out in accordance with the approved guidelines.

Provenance and peer review Not commissioned; externally peer reviewed.

Data availability statement Data are available upon reasonable request. Deidentified participant data are available upon reasonable request.

ORCID iD

Ho-Jin Lee http://orcid.org/0000-0002-7134-5044

\section{REFERENCES}

1 Werner MU, Kongsgaard UE. I. defining persistent post-surgical pain: is an update required? Br J Anaesth 2014;113:1-4.

2 Thapa P, Euasobhon P. Chronic postsurgical pain: current evidence for prevention and management. Korean J Pain 2018:31:155-73.

3 Glare P, Aubrey KR, Myles PS. Transition from acute to chronic pain after surgery. Lancet 2019:393:1537-46.

4 Kehlet $\mathrm{H}$, Jensen TS, Woolf CJ. Persistent postsurgical pain: risk factors and prevention. Lancet 2006;367:1618-25.

5 Gan TJ. Poorly controlled postoperative pain: prevalence, consequences, and prevention. J Pain Res 2017;10:2287-98.

6 Brummett CM, Waljee JF, Goesling J, et al. New persistent opioid use after minor and major surgical procedures in US adults. JAMA Surg 2017;152:e170504.

7 Bayman EO, Brennan TJ. Incidence and severity of chronic pain at 3 and 6 months after thoracotomy: meta-analysis. J Pain 2014;15:887-97.

8 Bayman EO, Parekh KR, Keech J, et al. A prospective study of chronic pain after thoracic surgery. Anesthesiology 2017;126:938-51.

9 Petersen $\mathrm{RH}$, Hansen $\mathrm{HJ}$, Dirksen $\mathrm{A}$, et al. Lung cancer screening and video-assisted thoracic surgery. J Thorac Oncol 2012;7:1026-31.

10 National Lung Screening Trial Research Team, Aberle DR, Adams AM, et al. Reduced lung-cancer mortality with low-dose computed tomographic screening. N Eng/ J Med 2011;365:395-409.
11 Brown MR, Ramirez JD, Farquhar-Smith P. Pain in cancer survivors. Br J Pain 2014:8:139-53.

12 Clarke H, Soneji N, Ko DT, et al. Rates and risk factors for prolonged opioid use after major surgery: population based cohort study. BMJ 2014;348:g1251.

13 Fletcher D, Stamer UM, Pogatzki-Zahn E, et al. Chronic postsurgical pain in Europe: an observational study. Eur J Anaesthesiol 2015;32:725-34.

14 Bendixen M, Jørgensen OD, Kronborg C, et al. Postoperative pain and quality of life after lobectomy via video-assisted thoracoscopic surgery or anterolateral thoracotomy for early stage lung cancer: a randomised controlled trial. Lancet Oncol 2016:17:836-44.

15 Sugiyama Y, lida H, Amaya F, et al. Prevalence of chronic postsurgical pain after thoracotomy and total knee arthroplasty: a retrospective multicenter study in Japan (Japanese Study group of subacute postoperative pain). J Anesth 2018;32:434-8.

16 Maguire MF, Ravenscroft A, Beggs $D$, et al. A questionnaire study investigating the prevalence of the neuropathic component of chronic pain after thoracic surgery. Eur J Cardiothorac Surg 2006;29:800-5.

17 Peng Z, Li H, Zhang C, et al. A retrospective study of chronic post-surgical pain following thoracic surgery: prevalence, risk factors, incidence of neuropathic component, and impact on qualify of life. PLoS One 2014:9:e90014.

18 Mejdahl MK, Andersen KG, Gärtner R, et al. Persistent pain and sensory disturbances after treatment for breast cancer: six year nationwide follow-up study. BMJ 2013;346:f1865.

19 Schott GD. Delayed onset and resolution of pain: some observations and implications. Brain 2001;124:1067-76.

20 Velnar T, Bailey T, Smrkolj V. The wound healing process: an overview of the cellular and molecular mechanisms. J Int Med Res 2009;37:1528-42.

21 Strassels SA, McNicol E, Suleman R. Postoperative pain management: a practical review, part 2. Am J Health Syst Pharm 2005;62:2019-25.

22 Brat GA, Agniel D, Beam A, et al. Postsurgical prescriptions for opioid naive patients and association with overdose and misuse: retrospective cohort study. BMJ 2018:360: 5790

23 Oh TK, Jeon Y-T, Choi JW. Trends in chronic opioid use and association with five-year survival in South Korea: a population-based cohort study. $\mathrm{Br} J$ Anaesth 2019:123:655-63.

24 Berterame S, Erthal J, Thomas J, et al. Use of and barriers to access to opioid analgesics: a worldwide, regional, and national study. Lancet 2016:387:1644-56.

25 Reddi D. Preventing chronic postoperative pain. Anaesthesia 2016;71:64-71.

26 Rizk NP, Ghanie A, Hsu M, et al. A prospective trial comparing pain and quality of life measures after anatomic lung resection using thoracoscopy or thoracotomy. Ann Thorac Surg 2014:98:1160-6.

27 Roth RS, Qi J, Hamill JB, et al. Is chronic postsurgical pain surgery-induced? A study of persistent postoperative pain following breast reconstruction. Breast 2018:37:119-25.

28 Haroutiunian S, Nikolajsen L, Finnerup NB, et al. The neuropathic component in persistent postsurgical pain: a systematic literature review. Pain 2013;154:95-102.

29 Wildgaard K, Ringsted TK, Hansen HJ, et al. Persistent postsurgical pain after video-assisted thoracic surgery--an observational study. Acta Anaesthesio/ Scand 2016;60:650-8.

30 Choi $\mathrm{H}$, Lee E. Market analysis of narcotic analgesics in Korea using HIRA claims data. J Korean Acad Manag Care Pharm 2015;4:31-7. 